\title{
AUFSATZ
}

\section{Ein „obskurer" Weimarer Verfassungsvater? Oder: Wie Hugo Preuß seinen Auftrag bekam und ihn nutzte}

\author{
Detlef Lehnert
}

\section{Die historiografische Ausgangslage}

Der geschichtliche Ablauf erschien seit langem geklärt: „Zum hundertsten Geburtstag von Hugo Preuss“ publizierte der frühere stellvertretende „Berliner Tageblatt“-Chefredakteur Ernst Feder (1881 - 1964) als einer der wenigen noch lebenden Zeitzeugen die in Fachkreisen bekannte Version, wie sein demokratischer Gesinnungsfreund „Schöpfer der Weimarer Verfassung" wurde:

„Am 14. November 1918 veröffentlichte Preuss im ,Berliner Tageblatt` nach einer Aussprache mit dessen Chefredakteur Theodor Wolff einen Artikel, der historische Bedeutung erlangen sollte. ... Noch am gleichen Tage erhielt er eine Einladung von Friedrich Ebert, der ihm im Auftrage der Volksbeauftragten den Posten eines Staatssekretärs des Innern anbot, damit er die Verfassung des jungen Volksstaats schaffe. Preuss besprach sich mit seinen Freunden. Als ersten besuchte er Paul Nathan, den langjährigen Mitarbeiter Theodor Barths, und bat um seinen Rat. Nathan empfahl anzunehmen unter der Bedingung, daß die Wahlen zur Nationalversammlung sofort ausgeschrieben, und daß außer Preuss auch andere Bürgerliche in die Regierung berufen würden. So sagte er denn in seiner Unterredung mit Ebert zu unter der Bedingung: ,Ich stehe und falle mit der Nationalversammlung. 'Eberts Antwort war kurz: ,Ich auch. “ 1

Diese präzise Darstellung ist wesentlich so auch bereits zeitgenössisch überliefert ${ }^{2}$ und bekräftigt eine demokratische Gründungserzählung der Weimarer Republik: Weil Hugo Preuß (1860 - 1925) mit seinem bekanntesten Zeitungsartikel ${ }^{3}$ einerseits das Bürgertum aus seiner Anhänglichkeit zum alten Obrigkeitsstaat in Richtung des Engagements für einen neuen Volksstaat aufscheuchen wollte, andererseits ein „verkehrter Obrigkeitsstaat“ fortgesetzter revolutionärer „Diktatur“ seinen ebenso vehementen Widerspruch fand, war er den Mehrheitssozialdemokraten politisch hilfreich. Überdies hatte die SPD, wegen staatlicher Diskriminierung im Kaiserreich, selbst für solche Aufgaben fachlich „keine Leute“ ${ }^{4}$. Auch Preuß war zunächst die akademische Karriere im Kaiserreich verwehrt, was „seine entschieden

1 Ernst Feder, Der Schöpfer der Weimarer Verfassung, in: Bulletin des Presse- und Informationsamtes der Bundesregierung, Nr. 204 vom 28. Oktober 1960, S. 1963 - 1966, S. 1964.

2 Vgl. Ernst Feder, Hugo Preuß. Ein Lebensbild, Berlin 1926.

3 Vgl. Hugo Preuß, Volksstaat oder verkehrter Obrigkeitsstaat?, in: Berliner Tageblatt vom 14. November 1918; auch ders., Volksstaat oder verkehrter Obrigkeitsstaat? (1918), in: ders., Gesammelte Schriften, Bd. 4: Politik und Verfassung in der Weimarer Republik, herausgegeben von Detlef Lehnert, Tübingen 2008, S. $73-75$.

4 Damit wird Ebert von Preuß zitiert nach Theodor Wolff, Tagebücher 1914 - 1919, herausgegeben von Bernd Sösemann, Erster Teil, Boppard am Rhein 1984, S. 654. Ähnlich unter Berufung auf eine persönliche Mitteilung von Else Preuß bei Wilhelm Ziegler, Die deutsche Nationalversammlung 1919/20 und ihr Verfassungswerk, Berlin 1932, S. 364 / Anmerkung 18: „Wir sind nicht in der Lage, sie zu machen. Wenn Sie nicht bereit sind, müßte ich mich an andere wenden." 
demokratische Gesinnung“ ebenso mit sich brachte wie „seine Zugehörigkeit zum Judentum“, mit dem Ergebnis: „Die einzige Auszeichnung, die ihm die Berliner Universität verlieh, war ein disziplinarischer Verweis, nicht wegen seiner akademischen Tätigkeit, sondern wegen einer Rede als Berliner Stadtverordneter. "5 Aber die Berliner Handelshochschule, finanziert von der (zumeist liberalen und auch jüdischen) Kaufmannschaft, berief ihn schließlich 1906 zum Professor für Öffentliches Recht - und dann 1918, im absehbaren Scheitern des Obrigkeitsstaats, zu deren Rektor. Zuvor war Preuß 1910 unbesoldeter Berliner Stadtrat mit Zuständigkeit für das Verkehrswesen (somit ein Vorgänger von Ernst Reuter 1926) geworden und darin ein Impulsgeber der Bildung des (heutigen) Groß-Berlins. ${ }^{6}$ Zudem erschien er für eine situationsbedingt rasche Entwurfslieferung vorbereitet, weil er 1917 eine parlamentarisierende Verfassungsreform ausgearbeitet hatte. ${ }^{7}$ Ohnehin galt er im Übergang vom Kaiserreich zur Republik als der „wohl am weitesten ,links' gerichtete Staatsrechtslehrer des damaligen Deutschlands"8 und darin besser als zum Beispiel Max Weber mit dem Revolutionskabinett kompatibel. Auch die USPD-Volksbeauftragten haben der Ernennung dieses standfesten bürgerlichen Demokraten offenbar nicht widersprochen. ${ }^{9}$

\section{Ergänzungsfundstücke I: Von wem der Vorschlag kam und wer noch mitwirkte}

Dieses skizzierte Geschichtsbild ist nicht falsch, aber unvollständig. Bislang gab es nur den fast unbeachteten Hinweis, dass der auf Kommunalfragen fokussierte und so mit Preuß bekannte SPD-Reichstagsabgeordnete Albert Südekum diesen Vorschlag an Ebert herangetragen haben soll. ${ }^{10}$ In der mittlerweile vorliegenden Biografie Südekums findet sich jedoch dazu nichts. ${ }^{11}$ In dem von Philipp Scheidemann (1865 - 1939), der am 9. November 1918 die Republik ausrief, verfassten Nachruf ist aber diese ergänzende Information zum Ursprung des Verfassungsauftrags für Preuß enthalten: „Landsberg ist es zu danken, daß er auf Preuß aufmerksam gemacht hat. Ebert, der Preußbis dahin überhaupt nicht kannte, war ohne weiteres einverstanden, mit Preuß Rücksprache zu nehmen und ihn zu berufen. "12 Das

5 Ernst Feder, a.a.O. (Fn. 1), S. 1963 f.; zum Anlass die Einleitung und die Texte in: Hugo Preuß, Gesammelte Schriften. Bd. 5: Kommunalwissenschaft und Kommunalpolitik, herausgegeben von Christoph Müller, Tübingen 2012 (im Erscheinen).

$6 \mathrm{Zu}$ Kommunalthemen vgl. Siegfried Grassmann, Hugo Preuß und die deutsche Selbstverwaltung, Lübeck 1964; Detlef Lehnert, Kommunale Institutionen zwischen Honoratiorenverwaltung und Massendemokratie, Baden-Baden 1994; Sandro Mezzadra, La costituzione del sociale. Il pensiero politico e giuridico di Hugo Preuss, Bologna 1999.

7 Vgl. Hugo Preuß, Vorschläge zur Abänderung der Reichsverfassung und der Preußischen Verfassung, nebst Begründung, in: ders., Staat, Recht und Freiheit, Tübingen 1926 (Neudruck Hildesheim 2006), S. $290-335$.

8 Walter Jellinek, Entstehung und Ausbau der Weimarer Reichsverfassung, in: Gerhard Anschütz I Richard Thoma (Hrsg.), Handbuch des Deutschen Staatsrechts. Bd. 1, Tübingen 1930, S. 127 138, S. 127.

9 Vgl. Protokoll der Kabinettssitzung vom 15. November 1918, in: Die Regierung der Volksbeauftragten 1918/19, Bearbeiter Susanne Miller / Heinrich Potthoff, Teil 1, Düsseldorf 1969, S. 40 f.

10 Vgl. Willibalt Apelt, Jurist im Wandel der Staatsformen, Tübingen 1965, S. 79; ders., Geschichte der Weimarer Verfassung, München 1946, wo er jedoch den Namen Südekum überhaupt nicht erwähnt.

11 Vgl. Max Bloch, Albert Südekum (1871 - 1944), Düsseldorf 2009.

12 Philipp Scheidemann, Hugo Preuß zum Gedächtnis, in: Vorwärts vom 13. Oktober 1925; ders., Der Zusammenbruch, Berlin 1921; ders., Memoiren eines Sozialdemokraten, 2 Bde., Dresden 
widerspricht der bislang einseitig hervorgehobenen Bedeutung des „Tageblatt“-Artikels nicht. Als Jurist wird der Volksbeauftragte Otto Landsberg (1869 - 1957), auch er jüdischer Herkunft, wohl bereits Schriften von Preuß gekannt und geschätzt haben. Von Landsberg gibt es bislang über die beruflichen und politischen Grundstationen hinaus so wenig einschlägiges biografisches Material wie zu Preuß oder auch für Ebert. ${ }^{13}$ Gerade weil Scheidemann kein eigenes Verdienst beanspruchte und Eberts Anteil an der Umsetzung der Anregung nicht verkleinerte, spricht nichts gegen diese Nachruf-Version. Darin standen zu Preuß ferner die anerkennenden Worte, dass er auch „einer der wenigen deutschen Bürger war, die sich vom Jahre 1916 ab über den voraussichtlichen Ausgang des Krieges nicht mehr den geringsten Täuschungen hingegeben haben“, und dann in der Funktion des Innenministers „Zweifellos der beste Mann, den die junge Republik in kritischster Stunde berufen konnte“.

Noch darüber hinaus wird der Tagesablauf in dem Erinnerungs-Artikel des mit Preu $\beta$ befreundeten Paul Nathan $\left(1857\right.$ - 1927) ${ }^{14}$ präzisiert. Dieser war - mit ähnlicher Kritik an der DDP wie Preuß, aber insofern anderer Schlussfolgerung - 1921 zur SPD übergetreten. Vielleicht auch deshalb drückte sich Nathan teils politisch etwas allgemeiner ${ }^{15}$, teils jedoch verlaufsgenauer über seine Reaktion auf einen Besuch von Preuß mit der dringlichen Frage nach der Ernennungszusage aus:

„Natürlich, wenn Ihnen freie Hand für die Schaffung einer demokratischen Verfassung garantiert wird. ' Eine halbe Stunde später waren wir bei Theodor Wolff vom ,Berliner Tageblatt', unmittelbar darauf traf auch dort noch Witting, der frühere Oberbürgermeister von Posen, ein, und wir alle, die Preuß zusammengerufen hatten, waren einig, daß Preuß seine Zusage Ebert geben müßte, die nötige Unabhängigkeit der Bewegung vorausgesetzt. Und so fuhr Preuß von der Jerusalemer Straße nach der Wilhelmstraße zum Sitze der provisorischen Regierung, und als er Ebert verließ, gab es in Deutschland den neuen Reichsminister des Innern, der den Auftrag hatte, der Republik Deutschland das Grundgesetz zu schaffen."16

1928, sind dazu weniger ergiebig. Als Leitfaden einer Würdigung: Christian Gellinek, Philipp Scheidemann. Gedächtnis und Erinnerung, Münster 2006.

13 Die monumentale Biografie von Walter Mühlhausen, Friedrich Ebert 1871 - 1925. Reichspräsident der Weimarer Republik, Bonn 2006, enthält zur Ernennung von Preußnichts außer dem Hinweis auf die - auch von Scheidemann bestätigte - bei Ebert liegende Verhandlungsführung (S. 123). Ein der „politischen Klarheit des Genossen Haase “ im sechsköpfigen Revolutionskabinett vertrauendes USPD-Blatt äußerte sich zu den MSPD-Volksbeauftragten differenziert: „Ebert ist ein fähiger und rücksichtsloser Herr. Möge er diese Eigenschaften im Kampf gegen die Volksfeinde verwenden. Von Scheidemann erwarten wir nichts, dagegen ist Landsberg ein hervorragend kluger Kopf mit einem Europäergewissen“; Bergische Arbeiterstimme vom 12. November 1919 (Artikel: „Am Tage nach der Revolution").

14 Im Hause Nathans gab eine „Freundesecke“ mit unter anderem einem Foto von Preuß; vgl. Ernst Feder, Politik und Humanität: Paul Nathan. Ein Lebensbild, Berlin 1929, S. 136 f.

15 Bei Günther Gillessen, Hugo Preuß. Studien zur Ideen- und Verfassungsgeschichte der Weimarer Republik, Berlin 2000 (Dissertation 1955), S. 104 (mit Anmerkung 4) ist aus - 1955 noch möglichen - Gesprächen mit seinem Sohn Dr. Ernst Preuß (London) die Bedingung (Einberufung der Nationalversammlung und weitere bürgerlich-liberale Fachleute) überliefert. Dort auch der Hinweis, dass „der gesamte persönliche Nachlaß Hugo Preuß vermutlich bei der Emigration der Söhne nach 1933 oder während des Luftkrieges in Berlin verloren gegangen ist“ (S. 9), bis auf minimale Reste (vgl. Anmerkung 17).

16 Paul Nathan, Hugo Preuß, in: Vorwärts vom 9. Oktober 1925. Auch bei Theodor Wolff, a.a.O. (Fn. 4), S. 654 sind diese Anwesenden bestätigt mit zusätzlichem Hinweis auf Alfred Weber. 
Erwähnenswert ist nicht allein, dass sich der als selbstbewusst bekannte Preuß derart beraten ließ, sondern der tatsächlich so aus weiteren Quellen zu bestätigende engste Kreis. In einem der wenigen überlieferten Briefe an Richard Witting hatte Preußam 12. Juli 1917 mitgeteilt, er habe sich nun insofern „zu Ihrer Meinung bekehrt, als ich die für alle Fälle nützliche Durcharbeitung der schliesslich einmal vorzunehmenden Verfassungsänderungen schon jetzt als erspriesslich einsehe“; überdies sei das Friedensthema nur „mit einer wirklichen, durch sich selbst überzeugenden inneren Umgestaltung " erfolgversprechend anzupacken. ${ }^{17}$ Entgegen Legenden, dass Preuß mit einem Verfassungsentwurf in der Tasche sozusagen auf seine Freiherr vom Stein-Stunde in einer Staatskrise gewartet haben könnte beziehungsweise der Kreis um Erich Ludendorff ausgerechnet ihn als Berater gewinnen wollte, ist es wohl so gewesen: Aus seinen Jahren als nationalliberaler Oberbürgermeister hatte Witting noch Kontakte zu Beratern an der Staatsspitze. Dabei wusste er noch nicht, dass Paul von Hindenburg und Ludendorff ultimativ die Trennung Kaiser Wilhelms II. vom Reichskanzler Theobald von Bethmann Hollweg erzwangen, der ihnen zu nachgiebig gegenüber Reichstagwünschen erschien. ${ }^{18}$ So konnte er Preuß gegen dessen Überzeugung, dass zunächst eine Verschiebung der Machtverhältnisse zugunsten des Reichstags absehbar sein müsse, als Verfasser eines Reformentwurfs gewinnen. Ein Primat der Innenpolitik folgte bereits aus der 1915 publizierten Schrift „Das deutsche Volk und die Politik“19. Dabei war Preuß, seine früheren Gedanken zum Bedarf an einem Völkerrecht ${ }^{20}$ weiterführend, allenfalls von friedensstiftender Kraft einer Internationale der modernen Demokratien überzeugt.

\section{Ergänzungsfundstücke II: Richtungswechsel im Beraterkreis}

Aber nicht nur Preuß war hier zur Mitwirkung, sondern zuvor bereits der ehemalige Nationalliberale Richard Witting (1856 - 1923) schrittweise nach links hin bekehrt worden ${ }^{21}$ : Er war „durch den Kriegstod eines Sohnes leidenschaftlicher Pazifist geworden; das alte Mitglied des Herrenhauses hatte sich, schwer enttäuscht von der Schlaffheit seiner Deutschen Demokratischen Partei, wenigstens innerlich bis dicht an die U.S.P.D. heran entwickelt" ${ }^{\text {"22 }}$. Noch bedeutsamer als diese spätere Auskunft ist ein Anfang Januar 1919 unter seinem Pseudonym Georg Metzler (damit wohl auf das Kriegsgemetzel anspielend) veröffentlichter

17 Preuß an Witting am 12. Juli 1917, Bundesarchiv Berlin-Lichterfelde, NL Preuß 90 Pr I, Bd. 1, B1. 69.

18 Vgl. Wolfram Pyta, Hindenburg. Herrschaft zwischen Hohenzollern und Hitler, München 2007, S. 277 - 283, S. 942 / Anmerkung 138.

19 Vgl. Hugo Preuß, Das deutsche Volk und die Politik (1915), in: ders., Gesammelte Schriften, Bd. 1: Politik und Gesellschaft im Kaiserreich, herausgegeben von Lothar Albertin, Tübingen 2007, S. $383-530$.

20 Vgl. ders., Das Völkerrecht im Dienste des Wirtschaftslebens (1891), in: ders., Gesammelte Schriften, Bd. 2: Öffentliches Recht und Rechtsphilosophie im Kaiserreich, herausgegeben von Dian Schefold, Tübingen 2009, S. 426 - 467.

$21 \mathrm{Zu}$ seiner Person gibt es kaum historische Studien, einige Stichworte dazu bei Arthur Kronthal, Witting, Richard, in: Deutsches Biographisches Jahrbuch, Bd. V: Das Jahr 1923, Berlin 1930, S. $395-403$.

22 Richard Witting, Brief an den Herausgeber, in: Die Weltbühne vom 3. Januar 1924, S. 17 (Nachruf des Herausgebers Siegfried Jacobsohn). 
Artikel $^{23}$, der in Geschichtsfälschungen zu Ursachen der Kriegsniederlage einen Hauptgrund der Gefährdung des republikanischen Neubeginns sah: „Vier Jahre lang ist das deutsche Volk morgens, mittags und abends über Ursache, Ursprung und Führung des Krieges belogen und betrogen worden." Auch in der SPD-Führung sah er durch zu langes Mittragen mancher Kriegslegende ein Hemmnis des Neuanfangs, weshalb er auf Erneuerung der gesamten demokratischen Linken hoffte: „Findet die Sozialdemokratie unkompromittierte Männer als Führer, so könnten diese mit ebenfalls unkompromittierten Persönlichkeiten aus der bürgerlichen Demokratie zusammen eine wirklich aktionsfähige Regierung bilden, die Ansehen und Glauben im Ausland und im Inland fände." Das war die bald „Weltbühne“typische Sicht eines von bitteren Erfahrungen geprägten Individualisten, der über Preuß Reformeifer noch hinaus greifend an den organisierten Kräften vorbei sich die politische Welt ganz neu denken wollte.

Eine zumindest phasenweise enge Verbindung zu Theodor Wolff(1868 - 1943) ist ebenfalls aus einem Zeitdokument über Preuß belegt: „Und schon am zehnten November war sich Theodor Wolff mit ihm über die Zertrümmerung des alten wasserstiefelnden Freisinns und der Begründung einer republikanisch-demokratischen Partei einig geworden. In einem kleinen Konferenzzimmer des Berliner Tageblatts traten darauf zu endlosen Sitzungen die paar Männer zusammen, die der neuen Partei den Lebensodem einbliesen. "24 Gewiss war der gesamte DDP-Gründerkreis weiter gezogen, aber für das sehr frühe diesbezügliche Engagement von Preuß und Witting bei Wolff gibt es weitere Hinweise. ${ }^{25}$ Eine Briefnotiz des wegen seiner imperialistischen Vergangenheit nicht zum Gründerkreis hinzugezogenen Friedrich Naumann liefert die bestätigende Gegenprobe: „Es handelt sich um eine Art Staatsstreich, der vom ,Berliner Tageblatt" ausgeht, man hat uns bolschewisiert. " ${ }^{26}$ Er meinte gewiss nur die - auch den Zeitumständen geschuldete - halb konspirative Methode der Gründungsinitiative, zentriert um ein publizistisches Flaggschiff des entschiedenen Linksliberalismus. Diese Vorgehensweise konnte führende Mitglieder der Vorkriegsparteien im Handstreich ohne Befragung gewissermaßen zu „Menschewiki“ (Minderheitlern) degradieren.

\section{Ergänzungsfundstücke III: Transatlantische Gesprächsnotizen}

Unscheinbare Fußnotenhinweise aus einer schon älteren, jedoch wegen der sorgfältigen Quellennähe noch immer lesenswerten Studie ${ }^{27}$ über die „Politik der Staatssekretäre“ (faktisch Ressortminister) des Kabinetts der Volksbeauftragten 1918/19 führen nun auf weitere

23 Georg Metzler (= Richard Witting), Die verruchte Lüge, in: Die Weltbühne vom 9. Januar 1919, S. 33, S. 35.

24 Johannes Fischart (= Erich Dombrowski), Hugo Preuß, in: Die Weltbühne vom 6. Februar 1919, S. 132.

25 Vgl. Lothar Albertin, Liberalismus und Demokratie am Anfang der Weimarer Republik. Eine vergleichende Analyse der Deutschen Demokratischen Partei und der Deutschen Volkspartei, Düsseldorf 1972, S. 56.

26 Naumann an Hohmann vom 25. November 1918, zitiert nach: Peter Theiner, Sozialer Liberalismus und deutsche Weltpolitik. Friedrich Naumann im Wilhelminischen Deutschland (1860 - 1919), Baden-Baden 1983, S. 288.

$27 \mathrm{Zu}$ Preuß vgl. Wolfgang Elben, Das Problem der Kontinuität in der deutschen Revolution. Die Politik der Staatssekretäre und der militärischen Führung vom November 1918 bis Februar 1919, Düsseldorf 1965, S. $45-69$. 
Spuren. Eine Gesprächsnotiz vom 2. Januar 1919, also unmittelbar vor der Veröffentlichung des ersten durchgearbeiteten Entwurfs der Weimarer Verfassung samt begleitender Denkschrift $^{28}$, liefert Einblicke in hintergründige Motive von Preuß. Der Chronist Ellis Loring Dresel (1865 - 1925), mit deutschen Vorfahren und so ohne Verständigungsprobleme, hat erstmals nach Kriegsende transatlantische Berichte solcher Intensität verfasst. ${ }^{29}$ Als hochrangiger Diplomat - vormaliger US-Attaché unter anderem in Berlin und dann zuständig für die Pariser Friedenskonferenzen - sowie ausgebildeter Jurist konnte er Gesprächsnotizen hinterlassen, die trotz Ungenauigkeiten in Einzelfällen ${ }^{30}$ urteilsfähig erscheinen.

So vermittelte Friedrich Ebert dem Besucher "the impression of an honest and simple man with the best intentions to build up the nation“; er habe aber wenig Sorge um eine Gefahr von rechts gezeigt: „Ebert said that he considered a reaction out of the question." Durchaus schon zur Jahreswende 1918/19 - und so als unausgesprochener Nebengedanke der starken Präsidialkomponente im Verfassungsentwurf - absehbar erschien der Zugriff Eberts auf das höchste Staatsamt: „He is everywhere spoken of as the logical President for the new German Republic. "31 Von Theodor Wolff erfuhr der US-Interviewer höchst beruhigende Proportionen: Etwa 400.000 sozialdemokratischen Demonstranten für republikanische Ziele und circa 100.000 aus demokratischem Bürgertum hätten kürzlich nur rund 30.000 Spartakisten gegenübergestanden. ${ }^{32}$ Auch Preuß bekundete gewisse Sorgen „from the Left“ nur vorübergehend in möglicher Folge von zugespitzter ökonomischer Not. Er äußerte sich aber zu Karl Liebknecht, dass dieser „not bloodthirsty“ sei, vielmehr „attented his courses, and had been an intelligent pupil“" (er studierte Anfang der 1890er Jahre in Berlin also Recht auch beim Privatdozenten Preuß), ,and that Rosa Luxemburg was the cleverest woman as far as politics were concerned in Germany today“. Jenseits des noch zu erörternden Weimarer Modells des parlamentarischen Regierungssystems inklusive präsidialer Exekutivspitze und Bundesstaatlichkeit ohne preußische Hegemonie konnten diese Informationen, Aussagen und Eindrükke vermerkt werden ${ }^{33}$ :

„Preuss was formerly a professor, and was considered the most radical member of the bourgeois parties. He was respected but obscure, and had no preferment until the new popular administration entered upon office ..., and was told that he was chosen because he was an independent thinker and because of this technical knowledge ...

To him the greater danger appeared to be from the Right, not for the present but in the long run, for he believed the reaction which was not showing its head now would emerge sooner or later and would emphasize any lack of success in any field. ...

28 Vgl. Hugo Preuß, Denkschrift zum Entwurf des allgemeinen Teils der Reichsverfassung vom 3. Januar 1919, in: ders., a.a.O. (Fn. 7), S. 368 - 394. Die Verfassungsentwürfe sind unter anderem abgedruckt bei Heinrich Triepel, Quellensammlung zum Deutschen Reichsstaatsrecht, Tübingen 1926.

29 Vgl. Manfred Jonas, The United States and Germany: A Diplomatic History, Ithaca 1984, S. 141.

30 So ist der Vorname von Preuß mit „G. F.“ verzeichnet, wohl schlicht ein Übertragungsfehler vom Manuskript.

31 Zitiert nach: „The Chief of the Special Mission in Germany (Dresel) to the Secretary of the Commission to Negotiate Peace (Grew)", in: Papers Relating to the Foreign Relations of the United States 1919. The Paris Peace Conference, (Department of State Publications 1823), Washington 1942, S. 164.

32 Vgl. ebenda, S. 153.

33 Vgl. die Beiträge in Detlef Lehnert (Hrsg.), Hugo Preuß 1860 - 1925, Köln 2011. 
The people were so used to the Hohenzollerns and to militarism that it would at best be a difficult matter to destroy the sentiments entirely. In this respect Dr. Preuss expressed a different opinion from other informants.

... The difficulty, he said, with political development in Germany was that Germany had never had a revolution and that therefore it lacked the leaven necessary for active political development. The present revolution, he also said, was too bloodless and had not been energetic enough, nor had it affected a sufficient number of the people.

Dr. Preuss gave the impression of a sincere man of exceptional intellect and acquirements. He is of Jewish origin." ${ }^{34}$

An der Authentizität dieser Aufzeichnungen kann es wenig begründete Zweifel geben, mit Ausnahme der in vielen Fällen (bei Preuß eher nur hinsichtlich des Typus der Bundesstaatlichkeit) registrierten Hervorhebung des amerikanischen Vorbilds, was hier insofern nur summarisch vermerkt bleibt. Dabei wird auf deutscher Seite das Bemühen um den legendären „Wilson-Frieden“ und vielleicht zusätzlich die besondere Aufnahmebereitschaft des herkunftsbedingt geneigten Chronisten für deutsch-amerikanische Verbindungslinien eine Rolle gespielt haben. Das als „respected but obscure“ bezeichnete akademisch-politische Vorleben von Preuß könnte hier sinngerecht als „respektiert, doch aus dem Verborgenen kommend“ übersetzt werden. ${ }^{35}$

\section{Das schwierige Verhältnis zum Judentum und zur höchsten Souveränität}

Zwar fanden sich unter den erwähnten Persönlichkeiten fast ausschließlich Bürger jüdischer Herkunft - vom Rechtsanwalt und Volksbeauftragten Landsberg über den Chefredakteur Wolff und den Ex-Oberbürgermeister und Bankier Witting bis hin zum Publizisten Nathan. Dort kann aber von innerer Homogenität keineswegs die Rede sein: Vom dann protestantisch in die gouvernementale Herrschaftskaste aufgestiegenen Witting bis zu dem auch zionistisch engagierten Nathan - und dazwischen meist glaubensfern assimilierten Kulturmodernisten nach Art Wolffs und dem konfessionslosen Landsberg - entfaltete sich ein breites Spektrum. Nur Preuß selbst gehörte seit 1891 und dann 1911 bis 1918 sogar dem Vorstand jener elitären „Gesellschaft der Freunde“ an, die sich als ursprüngliche jüdische Aufklärungsgesellschaft jedoch im Sinne des Grundsatzes, „dass Religion Privatangelegenheit der Mitglieder sei und den Verein nicht zu interessieren habe“, vom Konfessionalismus bewusst fernhielt. ${ }^{36}$ Dort noch länger Vorstandsmitglied (1904 - 1920) war unter den auch kulturell Prominenten nur der Verleger Rudolf Mosse; als Mitglieder finden sich aber unter anderem der Maler Max Liebermann sowie die Industriellendynastie Rathenau und die Verlegerfamilie Ullstein. Man wollte offenbar keine gesellschaftliche Spaltung zwischen gläubig oder nur formell jüdischen

34 „The Chief of the Special Mission in Germany (Dresel) to the Secretary of the Commission to Negotiate Peace (Grew)", in: Papers Relating to the Foreign Relations of the United States 1919. The Paris Peace Conference, (Department of State Publications 1823), Washington 1942, S. $159 \mathrm{f}$.

35 Der Titel dieses Beitrags spielt begrifflich zugleich auf jene satirischen „Dunkelmännerbriefe“ an, mit denen Preuß, a.a.O. (Fn. 19), S. 338 - 354, das konservative bis reaktionäre Gegenmilieu des Kaiserreichs verspottete.

36 Sebastian Panwitz, Die Gesellschaft der Freunde (1792 - 1935), Hildesheim 2007, S. 51, dort zu Preuß, S. 162, S. 178, S. 260. Eine alphabetisch geordnete Mitgliederliste findet sich unter http:// www.gesellschaftderfreunde.de. 
beziehungsweise aus dieser Religionsgemeinschaft aus- oder zu einer anderen Konfession übergetretenen Mitgliedern erzeugen. Da weder seine frühen akademischen Vorbilder Otto von Gierke und Rudolf von Gneist noch sein politischer Mentor Theodor Barth jüdischer Herkunft waren, suchte nicht Preuß ein vorrangig jüdisches Umfeld, sondern es hat sich von der Handelshochschule bis zum Beraterkreis des Verfassungsauftrags auf seiner akademisch-politischen Erfolgsleiter wegen anderweitiger Distanzverhältnisse so ergeben.

Über das innere Verhältnis zum Judentum ist bei Preuß fast nichts bekannt ${ }^{37}$, wie auch sonst zum privaten Bereich. Außer dass er persönlich wohl „uneitel“38 gewesen sei, wird nur sehr häufig sein Humor betont. Als Soldaten beim Erscheinen des Innenministers Preuß nicht „Haltung“ annahmen und sein Mitarbeiter Willibalt Apelt dies mit „So etwas wäre früher nicht möglich gewesen" kommentierte, erwiderte dessen Erinnerung nach Preuß: „Lassen Sie nur gut sein. Früher wäre ich auch nicht Reichsminister geworden. "39 Bei Theodor Heuss ist überliefert, dass Preuß sein "Judentum weder pathetisch noch sentimental“ auffasste, ohne je „seine Abstammung verheimlichen zu wollen“; es hat ihn „gar nicht sehr interessiert" hinsichtlich der „religiösen und traditionalen Seite“ ${ }^{40}$. Der Nachruf, den ihm ein „Jüdisches Jahrbuch“ seiner Heimatstadt widmete, bestätigt diese mangels tieferer Einblicke kursierenden Annahmen: „Bis in die letzten Lebensjahre blieb er dem öffentlichen jüdischen Leben fern. “ Zuletzt war er immerhin unter anderem neben Albert Einstein „zwei Jahre hindurch als stellvertretender Vorsitzender“ der „Akademie für die Wissenschaft des Judentums“ verbunden - fand also dem bisherigen Kenntnisstand gemäß nur solchen intellektuellen Zugang.

Dennoch würdigte jener Nachruf seine gesellschaftliche Standfestigkeit: „Hugo Preuß gehörte zu den Persönlichkeiten, für die die Zugehörigkeit zur jüdischen Gemeinschaft eine Hemmung ihrer Laufbahn bedeutet, die aber jede Zumutung, sie durch Lösung zu erleichtern, stolz und entschieden ablehnen. " ${ }^{11}$ Das reformjüdische Umfeld machte das geringe konfessionelle Interesse dem prominenten Gemeinschaftsmitglied rückblickend zunächst nicht besonders zum Vorwurf, sondern hob seine politische Impulsgeberrolle hervor: „Hugo Preuß war Demokrat, lange ehe es die Deutsche Demokratische Partei gab; er war ein überzeugter Republikaner von jeher. “42 Letzteres galt für seine Betonung der „,republikanischen

37 Vgl. Dian Schefold, Hugo Preuß: „Aus dem großen Zusammenbruch den demokratischen Volksstaat retten“, in: Hans Erler / Ernst Ludwig Ehrlich / Ludger Heid (Hrsg.), „Meinetwegen ist die Welt erschaffen“. Das intellektuelle Vermächtnis des deutschsprachigen Judentums, Frankfurt am Main 1977, S. 293 - 309; ders., Hugo Preuß (1860 - 1925), in: ders., Bewahrung der Demokratie, Berlin 2012, S. $151-174$.

38 So Theodor Heuss in seiner Gedenkrede an der Freien Universität Berlin zum 100. Todestag, zitiert nach: „Erhalter der Einheit“, in: Der Tagesspiegel vom 29. Oktober 1960.

39 Willibalt Apelt, Jurist, a.a.O. (Fn. 10), S. 95.

40 Theodor Heuss, Einleitung, in: Hugo Preuß, a.a.O. (Fn. 7), S. 1 - 23, S. 15.

41 Nachbemerkung zu R(einhold) Issberner, Hugo Preuß, in: Jüdisches Jahrbuch für Groß-Berlin auf das Jahr 1926, herausgegeben von Jacob Jacabson / Jacob Segall, Berlin 1927, S. 176. Seine einzige dokumentierte große Rede vor spezifisch verbandsjüdischem Publikum findet sich in Preuß, a.a.O. (Fn. 3), S. 291 - 301 (aus dem Sterbejahr 1925), mit der selbstbewussten Haltung: „Wem soll ich mich assimilieren? Bin ich ein minderer Deutscher als irgendein anderer?", S. 298.

42 R(einhold) Issberner, a.a.O. (Fn. 41), S. 176. Als „scharfer Demokrat“ bereits in den Vorkriegsjahren mochte er gemäßigteren Liberalen zugleich noch als ein „demokratischer Eigenbrödler" erschienen sein, dessen historische Stunde aber mit dem Systemwechsel vom November 1918 gekommen war: Der Welthandel vom 10. Januar 1919. 
Natur städtischen Wesens“ 43 im Spannungsverhältnis zu dynastischen Obrigkeitsmonarchien. Gleich dem englischen Beispiel hätte er sich auch mit einer vollparlamentarisierten deutschen Reichsmonarchie arrangieren können; sie hätte für ihn keinen Widerspruch zur Grundidee des „républicanisme“ im Sinne Immanuel Kants dargestellt, womit er frühzeitig „den Gegensatz des Volksstaats zum Obrigkeitsstaat“" markierte. ${ }^{44}$

Für den auch DDP-aktiven Nachrufautor verband Preuß, in sich den Staatsmann und den Gelehrten“ 45 und war „,bis zuletzt der typische Vertreter der ,Jugend“ in dem Sinne, daß er Zeit seines Lebens für die Zukunft arbeitete, und daß er, unbeschwert von den Fesseln einer verknöcherten Tradition, neue schöpferische Ideen hervorbrachte “ 46 . Indem Preußjenseits seiner Neigung zum intensiven Gebrauch von Zitaten der literarischen Klassiker insofern ein akzentuierter Modernist war, konnte ihm die persönliche Verwurzelung in kirchlichen und religiösen Orthodoxien nicht einleuchten. Erst recht fehlte ihm die Bereitschaft zum Glauben an Obrigkeitsdynastien „von Gottes Gnaden“. So entsprang seine Kritik der Souveränitätslehren von Staatlichkeit auch der Analogie zu anstaltlich hierarchisierten Religionsgemeinschaften: „Die Souveränität der allein herrschenden Staatsgewalt ist gleich der Kirche nur von oben nach unten zu konstruieren. Doch die Souveränität der Staatsgewalt strebt unvermeidlich nach ihrem Urquell, dem persönlichen Souverän zurück. “ ${ }^{47}$ Die Souveränität in das eine Volk oder dessen eine parlamentarische Nationalrepräsentation zu verlagern, konnte für ihn solche Tendenz zur Machtkonzentration in den Händen nur einer Person nicht komplett brechen.

\section{Das schwierige Verhältnis zum Parlamentarismusmodell von Robert Redslob}

Neben der mit einem genossenschaftstheoretischen Staatsverständnis verbundenen Herkunft aus der Gierke-Schule gehört es zu den gängigen Erklärungsmustern der Preußschen Verfassungsentwürfe 1918/19, dass er Grundgedanken von Robert Redslob übernommen habe, um neben das Parlament einen vom Volk gewählten Präsidenten zu stellen und ihn mit dem Recht zum Appell an dieses Volk zur Parlamentsauflösung auszustatten. Unzweifelhaft richtig bleibt daran nur, dass vermutlich ab August 1918 die vergleichende Studie von Redslob für Interessierte verfügbar gewesen ist und dass seine normative Terminologie vom „echten“ und ,unechten“ Parlamentarismus in der Weimarer Verfassungsdebatte - auch bei Preu $\beta_{-}$ punktuell verwendet wurde. ${ }^{48}$ Entgegen dem einseitigen Bild, dass der in Rostock lehrende

43 Hugo Preuß, Die Entwicklung des deutschen Städtewesens, Leipzig 1906, S. 188 (kein Bd. 2 erschienen).

44 Ders., Nationaler Gegensatz und internationale Gemeinschaft (1918), in: ders., a.a.O. (Fn. 19), S. $706-718$, S. 712.

45 Diese Charakterisierung auch bei Ernest Hamburger, Hugo Preuß. Scholar and Statesman, in: Leo Baeck Institute, Year Book 20/1, London 1975, S. 179 - 206; ders., Juden im öffentlichen Leben Deutschlands, Tübingen 1968, behandelt die Zeit bis 1918, dort zu dem sonst bislang nur wenig beachteten langjährigen Reichstagsabgeordneten Landsberg: S. 509 - 515.

46 R(einhold) Issberner, a.a.O. (Fn. 41), S. 174.

47 Hugo Preuß, Selbstverwaltung, Gemeinde, Staat, Souveränität (1908), in: ders., a.a.O. (Fn. 20), S. $236-269$, S. 262 f.

48 Vgl. etwas anders nuanciert Manfred Friedrich, Plan des Regierungssystems für die deutsche Republik. Zur Lehre vom ,echten' und ,unechten' Parlamentarismus: Robert Redslob und Hugo Preuß, in: Detlef Lehnert / Christoph Müller (Hrsg.), Vom Untertanenverband zur Bürgergenossenschaft. Symposion zum 75. Todestag von Hugo Preuß am 9. Oktober 2000, Baden-Baden 2003, S. $189-201$. 
Elsässer Redslob in verklärter Sicht des britischen Parlamentarismus die negative Kontrastfolie zur kritisierten französischen Situation gesucht habe, bilanziert er diese betont differenziert $^{49}$ : Frankreich sei das "große constitutionelle Laboratorium Europas“, und es bleibe sein „Verdienst, daß es zusammen mit England der Schöpfer des modernen Staates gewesen ist“ (S. 186). Tatsächlich sind die ergänzenden Studien zur - England benachbart gesehenen belgischen Verfassung (S. 68 - 82) sowie dann auch noch zu Ungarn und Schweden (S. 82 - 105) nur knapp formuliert und wird die „beschränkte Monarchie der deutschen Länder“ (S. 1) als vergleichsuntauglich ausgesondert. Wohlbemerkt sah er vor Brechung des monarchischen Primats das Auflösungsrecht in kritischer Perspektive: „So ist die Auflösung in den deutschen Monarchien nicht ein Appell an das Volk als den Schiedsrichter des Streites, sondern eine Waffe, um den Widerstand des Unterhauses zu brechen." (S. 99 f.) Als ob er den fortgeschleppten Missbrauch geahnt hätte, der am Ende der Weimarer Republik mit analoger Motivlage betrieben wurde, hielt er nur bei hinreichenden Indizien geänderten Mehrheitswillens die einmalige Parlamentsauflösung für ein geeignetes demokratisches Mittel: „Ein Volk anzurufen, dessen Gegnerschaft man kennt, heißt einen Act der Unterdrückung versuchen." Insbesondere war das Ergebnis eines solchen Appells an das Wahlvolk dann unbedingt zu respektieren: „Der Souverän hat gesprochen ... Parlament und Staatshaupt haben sich dem Willen ihres Herrn zu beugen. Eine Fortsetzung des Kampfes ist ein Bruch des parlamentarischen Regimes." (S. 132)

Keinesfalls orientierte sich Redslob am klassischen Parlamentsideal nach der englischen Wahlreform von 1832, denn diese Periode habe „das parlamentarische Regime noch nicht begründet“, weil die relativ schmale "Wahlkörperschaft“ vom Repräsentativorgan „absorbiert“ wurde (S. 26). Gleich zu Beginn postulierte nämlich der Autor „die Prärogative der Wähler“ (S. 3) und so eine „Bindung der Gewalten“ (S. 7) statt nur deren Trennung, was erst zu einem „integralen parlamentarischen Regime“ (S. 19) in England hinführt. Dieses gründet sich auf die „Organisation wohldisciplinierter Parteien“ (S. 36). Mit der schon recht nahe an das allgemeine Männerstimmrecht vordringenden Wahlreform William Gladstones von 1884 sei endgültig vom „parlamentarischen Regime“ zu sprechen, „dessen Grundgedanke die höchste Schiedsgewalt des Volkes ist“ (S. 62). Jenseits dieser eben nicht am bloßen Konstitutionalismus orientierten demokratischen Tendenz war der Irrtum Redslobs historischer Art: Er hatte unzureichend erkannt, dass frühere Kronrechte eines parlamentsauflösenden Appells an das Wahlvolk kaum mehr rückholbar auf das Premierministeramt übergegangen waren, das gerade dadurch die von Redslob bemerkten Züge eines plebiszitär legitimierten persönlichen Regiments annahm.

Eine auch nur landesspezifisch generell parlamentarismuskritische Sicht lässt sich aus der skeptischen Analyse der französischen Verhältnisse bei Redslob nicht erkennen. Stattdessen wird klärend dargelegt, wie eine missbräuchliche Anwendung des im Verfassungsgesetz vom 25. Februar 1875 enthaltenen Rechts des Präsidenten, die Deputiertenkammer mit Zustimmung des Senats aufzulösen (S. 120), dieses Mittel schon 1877 diskreditierte:

„Man hat dem Marschall de Mac Mahon vorgeworfen, er habe die Auflösung mißbraucht. Diese Kritik ist begründet. Der Präsident wußte wohl, daß das Volk nicht auf seiner Seite war. Die Ergänzungswahlen, die Volksversammlungen, die Presse belehrten ihn, daß kein Gegensatz vorhanden war zwischen der Nation und ihren Repräsentanten, sondern daß er selbst

49 Die folgenden Seitenzahlen nach Robert Redslob, Die parlamentarische Regierung in ihrer wahren und in ihrer unechten Form, Tübingen 1918. 
allein stand. ... Aber weiter, dieser Aufösung folgte ein Act, der im vollen Sinn des Wortes ungesetzlich war: nämlich die Bildung des Ministeriums de Rochebouët, eine Maßregel, die in offenem Widerspruch mit dem bei den allgemeinen Wahlen ausgesprochenen Willen des Volkes stand.“ ( S. 132)

Seither entstand in Frankreich eine „Quasi-Souveränität des Parlaments“ (S. 134), in der Sicht des Autors eine Rückbildung zu früheren britischen Zuständen, als „die höchste Autorität ... noch nicht auf die Wählerschaft übergegangen“ war: „Die englische Verfassung nach 1832 zeigt eine auffallende Analogie mit dem heute in Frankreich herrschenden System.“ (S. 135) In England ließ sich mit den ,großen demokratischen Reformen von 1867 und 1884“ (S. 137) der Parlamentarismus über die absorptive Repräsentation ${ }^{50}$ hinaus zu seiner vollendeten Gestalt fortbilden. Da er Konzepte bevorzugte, „das Recht durch die politischen Kräfte zu erklären“, lag es Redslob fern, ein Patentrezept der Verfassungsreform anzubieten: „Wenn in den deutschen Monarchien die Kammern unfähig sind, ein Cabinett zu stürzen, wenn hingegen in Belgien, in England und in anderen Ländern eine Regierung nur lebensfähig ist kraft der Unterstützung durch das Parlament oder die Wähler, so hat diese verschiedene Constellation ihren Ursprung nicht in Verfassungsartikeln, sondern in den Traditionen und der Geistesverfassung des Volks." (S. 168 mit Anmerkung 2)

Der Autor empfahl nicht einfach die Volkswahl des Präsidenten nach amerikanischem Muster: „Es wäre gewagt, hier eine Lösung geben zu wollen.“ (S. 185) Eindeutig fiel allein seine Problemdiagnose aus, dass es „die Tendenz zur Einheit“ sei, die sich „dem wahren parlamentarischen Regime entgegenstellt“, was eben nicht souveräne Repräsentation an nur einer Stelle bedeute: „Diese Tendenz zur Einheit, zur Vereinfachung ... hindert Frankreich, ein System der Dualität einzuführen“, und verurteile „das Volk zum Schweigen ... außer alle vier Jahre bei den ordentlichen Wahlen“ (S. 180). Darin beklagte Redslob die mit der „unglücklichen Präcedens von 1877“ (S. 128) aus der Verfassungspraxis entschwundene Option der Auflösung des Parlaments zwecks Befragung der Wählerschaft in wichtigen Konfliktfällen. Daraus folgt zunächst nur, dass ein parlamentarisch gewählter Präsident, den auch Preuß zu Beginn seiner Tätigkeit zunächst präferiert haben soll ${ }^{51}$, dann eben allein in hinreichend eindeutigen Fällen der Abweichung einer Parlamentsmehrheit vom indiziell ersichtlichen klaren Mehrheitswillen der Wählerschaft das Auflösungsrecht einsetzen sollte.

Wenn Preuß ähnlich wie Redslob im französischen System die „Monokratie des Parlaments " 52 bemängelte, dann eben auch in Distanz zur absorptiven Repräsentation als nunmehr unvollständig erscheinende Demokratie. ${ }^{53}$ Überdies hatte er schon eine Generation zuvor sein Plädoyer für den Parlamentarismus, gegen Unmittelbarkeitsmythen einseitig

50 Als deren prominenter Vertreter in der Gegenwart: Ernst-Wolfgang Böckenförde, Mittelbare / repräsentative Demokratie als eigentliche Form der Demokratie, in: Georg Müller / René A. Rhinow / Gerhard Schmid / Luzius Wildhaber (Hrsg.), Staatsorganisation und Staatsfunktionen im Wandel. Festschrift für Kurt Eichenberger zum 60. Geburtstag, Basel 1982, S. $301-328$.

51 Vgl. Willibalt Apelt, Geschichte, a.a.O. (Fn. 10), S. 57, der zwar irrt, dass erst Max Weber in den Dezemberberatungen die Umstimmung erreichte, aber durchaus richtig erinnern könnte, dass Preußzu Beginn der Tätigkeit im November noch anders dachte.

52 Hugo Preuß, Begründung des Entwurfs einer Verfassung für das Deutsche Reich (1919), in: ders., a.a.O. (Fn. 7), S. $394-421$, S. 417.

53 Eine britische Studie hat Preußfür sein Konzept „good democratic reasons” bescheinigt: Peter Stirk, Hugo Preuss. German Political Thought and the Weimar Constitution, in: History of Political Thought, 23. Jg. (2002), H. 3, S. 497 - 516, S. 497 f., S. 514. 
direktdemokratischer Souveränitätslehren damit begründet, dass stets eine „Mehrzahl von Machtzentren“ vorhanden sein müsse, die erst zur „Möglichkeit ehrlichen Kampfes verschiedener Machtfaktoren“ führe: „Wo nur ein Machtzentrum existiert, gibt es keine Freiheit, sondern Despotie, gleichviel, welcher Art jenes sei. "54 Daraus folgte, dass Preuß 1918/19 neben einem Mehrebenen-Staat aus einem Stufenaufbau der Gebietskörperschaften nun auch eine Mehrebenen-Demokratie vorsah, die - zentriert um die Parlamentsregierung ergänzende plebiszitäre Komponenten aufwies. Es war lange vor der ungeklärten Frage intensiver oder nur situativ entlehnter Redslob-Rezeption sein aus der Genossenschaftstheorie folgendes pluralistisches Gesellschafts- und Verfassungsdenken ${ }^{55}$, das Preuß weder ein homogenisiertes Gesamtvolk, noch das Nationalparlament oder einen Staatsverband beziehungsweise gar Einzelpersonen mit Souveränitätsgewalt ausgestattet sehen wollte. Zwar verstand Max Weber den „vom Volke bestellten Präsidenten“ tatsächlich als „Gegengewicht gegen das Parlament“, aber Preuß plädierte nur für die Rechte wie bei einem „beschränkten parlamentarischen Monarchen“ und hatte so dem Reichskanzler „die Stellung eines englischen Premier-Ministers " zugedacht. ${ }^{56}$ Dort in Reserve gehaltene Kronrechte wird er kaum maßlos überschätzt haben; sein Denkfehler lag eher in den Grenzen einer Richtlinienkompetenz des Kanzlers (Art. 56 WRV bereits textanalog Art. 65 GG) bei notwendigen Koalitionsbildungen.

Dabei sollte aber nicht aus dem Blick geraten, wie sehr Preuß das Wirken historisch-politischer Kräfte deren Einfassung in Rechtsnormen vorgeordnet sah: „Die Reichsverfassung der Deutschen Republik vom 11. August 1919 ist der staatsrechtliche Niederschlag der Revolution vom 9. November 1918." 57 Mit Traditionsüberhang älterer konstitutioneller Gleichgewichtsdoktrinen hatte das wenig gemein. Preuß begründete sein Konzept der „parlamentarischen Demokratie“ ohnehin „weniger von Verfassungsparagraphen“ her als von der realen „wirtschaftlichen und sozialen Struktur der Gesellschaft“ .58 Von einer Gegenüberstellung des gesamten Parlaments zur Regierung wie im konstitutionalistischen Denken der Vorkriegszeit war bei Preußgerade nicht die Rede: „Das Wesen des Parlamentarismus beruht auf einer durch den Zusammenhang mit der Parlamentsmehrheit starken Regierung. "59 Er argumentierte statt aus liberalen Illusionen einer Balanceharmonie vielmehr unter dem Primat eines demokratischen Konfliktpluralismus:

54 Hugo Preuß, Die Sozialdemokratie und der Parlamentarismus (1891), in: ders., a.a.O. (Fn. 7), S. 144 - 172, S. 168 (auch: ders., a.a.O. (Fn. 19), S. 176 - 198, S. 195).

55 Vgl. Andreas Voßkuble, Hugo Preuß als Vordenker einer Verfassungstheorie des Pluralismus, in: Jürgen Kocka / Günter Stock (Hrsg.), Hugo Preuß: Vordenker der Pluralismustheorie, Berlin 2011, S. 23 - 42; Detlef Lehnert, Das pluralistische Staatsdenken von Hugo Preuß, Baden-Baden 2012 - jeweils mit weiterer neuerer Literatur.

56 Zitiert nach Max Weber, Gesamtausgabe, Bd. 16, herausgegeben von Wolfgang J. Mommsen, Tübingen 1988, S. 85 f., S. 89.

57 Hugo Preuß, Deutschlands Republikanische Reichsverfassung (1923), in: ders., a.a.O. (Fn. 3), S. $307-363$, S. 308.

58 Ders., Denkschrift zum Entwurf des allgemeinen Teils der Reichsverfassung vom 3. Januar 1919, in: ders., a.a.O. (Fn. 7), S. $368-394$, S. 386.

59 Ders., Deutschlands Staatsumwälzung (1919), in: ders., a.a.O. (Fn. 3), S. 101 - 113, S. 108. Kritik am insoweit falsch verstandenen „Dogma der Gewaltenteilung" auch schon in: ders., Die organische Bedeutung der Art. 15 und 17 der Reichsverfassung (1889), in: ders., a.a.O. (Fn. 20), S. $405-424$, S. $407 \mathrm{ff}$. 


\begin{abstract}
„Das Wertvollste an unserer Reichsverfassung ist die Elastizität in der Abgrenzung der Machtverhältnisse zwischen Reichspräsident, Reichsregierung und Reichstag. Bei dieser Elastizität wird sich die Macht immer dahin verlagern, wo die größte innere politische Energie ist. Unter diesem Gesichtspunkt muß der Reichspräsident gewählt werden. Unter diesem Gesichtspunkt ist es notwendig, daß das deutsche Volk bei der ersten Präsidentschaftswahl nicht einen Bock zum Gärtner mache."60
\end{abstract}

So wie in Frankreich erst der gescheiterte Übergriff Patrice de Mac Mahons 1877 das Parlament vor künftigen Präsidialappellen an das Volk abgeschirmt hatte, lag es mehr an historisch-politischen als verfassungsrechtlichen Faktoren, wenn in Deutschland dem mit Zweidrittel- beziehungsweise Dreiviertelmehrheit im Parlament bestellten, aber 54-jährig verstorbenen „Gärtner" der Republik Ebert dann ein nur mit knapper relativer Stimmenmehrheit gewählter 77 -jähriger kapitaler „Bock“ wie Hindenburg nachfolgte. ${ }^{61}$ Fatal im Vergleich zu der frühzeitig alarmierenden Situation 1877 in Frankreich wirkte sich aus, dass der Einsatz des Art. 48 WRV bis zum Krisenwinter 1923/24 und der Reichstagsauflösung im Herbst 1924 trotz etlicher Kritikpunkte von Preuß $5^{62}$ in der Absicht und Wirkung republikfreundlich gesehen wurde, daraufhin sogar Hindenburg in der Phase relativer Stabilisierung bis Herbst 1929 zurückhaltend agieren konnte, aber seine verfassungsfremden Handlungen in der tiefen Krise seit 1930 dann schleichend den Abschied von Weimar herbeiführten. ${ }^{63}$

\title{
7. Der schwierige Rückblick aus der Bundesrepublik
}

Die Erinnerung an Hugo Preuß blieb nach 1945 mit dem Geschichtsbild belastet, dass die Weimarer Verfassung die Katastrophe von 1933 mit verursacht habe. Das galt nicht für noch aus persönlicher Erinnerung geprägte Darstellungen wie jene des seinerzeit ebenfalls der DDP angehörenden Mitarbeiters Apelt: Er zählte Preußals „Vater der Weimarer Verfassung“ neben Ebert und den ermordeten Ministern Matthias Erzberger und Walther Rathenau zu den namhaftesten Politikern aus den Gründungsjahren der lange unterschätzten ersten deutschen Republik. ${ }^{64}$ Als jedoch der ehemalige Bundespräsident Theodor Heuss eine Feier zum

60 Ders., Preuß für Hellpach (1925), in: ders., a.a.O. (Fn. 3), S. 618 - 619, S. 619.

61 Das Sprachbild vom „Bock als Gärtner“ verwendete auch Hermann Heller in Replik auf Carl Schmitt im Preußenschlag-Prozess 1932 als letztlich naheliegend, „das Verhältnis gewisser Staatsrechtslehrer zur gegenwärtig geltenden Reichsverfassung so zu kennzeichnen“, zitiert nach Kathrin Groh, Demokratische Staatsrechtslehrer in der Weimarer Republik, Tübingen 2010, S. 141 / Anmerkung 2.

62 Dazu Belege in Detlef Lehnert, Einleitung, in: Hugo Preuß, a.a.O. (Fn. 3), S. 1 - 70, S. 35 f.

63 Sogar bei konservativen Staatsrechtslehrern wurde 1931 die Warnung formuliert, dass weitere Notverordnungspraxis unter Ausschaltung des Parlaments die verfassungswidrige „Grundlage für eine Dekretgesetzgebung würde, wie in dem vorfaschistischen Italien“; Erich Kaufmann, Gesammelte Schriften, Bd. 1, Göttingen 1960, S. 469. Im Wissen um die Folgen formulierte Willibalt Apelt, Geschichte, a.a.O. (Fn. 10), S. 379, noch pointierter, „daß in den letzten Jahren von Art. 48 ein Gebrauch gemacht wurde, der nur als Missbrauch bezeichnet werden kann. Der Ausnahmezustand wurde zu einem Dauerzustand."

64 Willibalt Apelt, Geschichte, a.a.O. (Fn. 10), S. 423 - 425. Seinem Zeugnis gemäß fand es auch Preuß als „unzulänglich, in einem Werk der demokratischen Erneuerung Deutschlands lediglich die allbekannten, vielfach abgegriffenen Gedanken liberaler Staatsauffassung des 19. Jahrhunderts 
100. Geburtstag von Preuß anregte, sprach sich der zuständige Bundesinnenminister Gerhard Schröder (von Kanzler Konrad Adenauer unwidersprochen) dagegen aus, „eine Verfassung, die das Grundgesetz habe überwinden wollen, durch eine staatliche Feier allzu stark herauszustellen“65. Der Titel „Berlin ehrt Hugo Preuss“ eines Berichts in der Frankfurter Allgemeinen Zeitung bezog sich auf die vom Senat der Stadt an der Freien Universität in Verbindung mit dem Deutschen Städtetag im Oktober 1960 durchgeführte Gedenkveranstaltung zum 100. Geburtstag von Preuß. Auf dieser sprachen neben dem Regierenden Bürgermeister Willy Brandt der Bundesminister für gesamtdeutsche Fragen Ernst Lemmer und als Hauptredner Bundespräsident a.D. Theodor Heuss. ${ }^{66}$

Unterschwellig spielte hier auch ein politischer Konflikt um historische Deutungsmacht hinein: Lemmer war vor 1933 Generalsekretär der liberalen Gewerkschaftsverbände und gehörte wie Heuss und Preuß als eines der prominenten DDP-Mitglieder auch dem Republikschutzbund Reichsbanner Schwarz Rot Gold an. Von diesem hatte sich der noch im späten Kaiserreich politisch sozialisierte Adenauer (der 22 Jahre vor Lemmer geboren war) im Unterschied zum linken Zentrumsflügel ferngehalten. Der wie Lemmer evangelische Innenminister Schröder war als Student am Ende der Weimarer Republik der „rechtsliberalen Deutschen Volkspartei (DVP)“ verbunden, bevor er 1933 NSDAP-Mitglied wurde. ${ }^{67}$ Mit der Unterstützung des Gedenkens durch Heuss und Lemmer sowie der Reserviertheit von Schröder verteidigten diese Politiker zugleich ihre politischen Biografien aus der Weimarer Zeit, auch wenn die Regierungsmitglieder anders als Heuss der CDU angehörten.

Diese kontroverse Erinnerungslage hat sich bis zum Festakt anlässlich des 150. Geburtstags von Hugo Preuß in der Berlin-Brandenburgischen Akademie der Wissenschaften im Jahre 2010 deutlich entspannt. ${ }^{68}$ Vielleicht besteht nun Hoffnung, dass im „kleinen“ Deutschland - entsprechend bescheidener - für den Verfassungsvater Preuß künftig noch gelingen möge, was im "großen“ Österreich mit einem Kelsen-Institut seinem Wiener Gegenstück der demokratischen Republikgründung von 1918 zu Ehren geschaffen wurde.

zu wiederholen“ (S. 296), glaubte jedoch anders als Naumann nicht an die Möglichkeit, die Aufgabe eines umfangreichen neuen Grundrechtskatalogs unter Zeitdruck bereits hinreichend bewältigen zu können.

65 Vgl. 114. Kabinettssitzung am 13. Juli 1960, zitiert nach http://www.bundesarchiv.de/cocoon/ barch/1000/k/k1960k/kap1_2/kap2_28/para3_20.html;jsessionid=11E6C1F0ECE6766EFBEC 140CDAAF02ED?highlight=true\&search=Hugo\%20Preu\%C3\%9F\&stemming=false\&field=all (Abruf am 27. November 2012).

66 FAZ vom 29. Oktober 1960. Kritisch zur Haltung der Bundesregierung Werner Stephan, Hugo Preuss, in: liberal, Nr. 4 / 1960, S. 23 - 26, S. 23: „In Bonn blieb man stumm. Der Geist, von dem der große Liberale erfüllt war, erschien den heute am Rhein Herrschenden denn doch gar zu fremd! “

67 Vgl. Stefan Marx / Wolfgang Tischner, Schröder, Gerhard, in: kas.de vom 30. Mai 2012, http:// www.kas.de/wf/de/71.8514 (Abruf am 27. November 2012); Torsten Oppelland, Gerhard Schröder (1910 - 1989), Düsseldorf 2002.

68 Vgl. diverse Beiträge in Jürgen Kocka / Günter Stock (Hrsg.), a.a.O. (Fn. 55). 